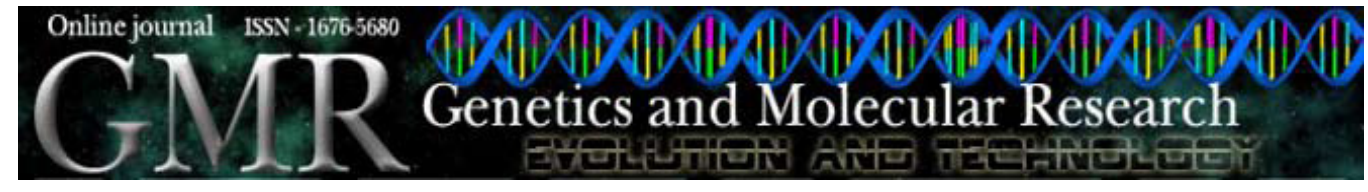

\title{
Normal and defective mariner-like elements in Rhynchosciara species (Sciaridae, Diptera)
}

\author{
P. Rezende-Teixeira, C. Lauand, F. Siviero and G.M. Machado-Santelli \\ Departamento de Biologia Celular e do Desenvolvimento, \\ Instituto de Ciências Biomédicas, \\ Universidade de São Paulo, São Paulo, SP, Brasil \\ Corresponding author: P. Rezende-Teixeira \\ E-mail: paularez@usp.br
}

Genet. Mol. Res. 9 (2): 849-857 (2010)

Received January 13, 2010

Accepted February 9, 2010

Published May 4, 2010

DOI 10.4238/vol9-2gmr796

\begin{abstract}
Mariner-like elements are widely present in diverse organisms. These elements constitute a large fraction of the eukaryotic genome; they transpose by a "cut-and-paste" mechanism with their own transposase protein. We found two groups of mobile elements in the genus Rhynchosciara. PCR using primers designed from $R$. americana transposons (Ramar1 and Ramar2) were the starting point for this comparative study. Genomic DNA templates of four species: R. hollaenderi, R. millerii, R. baschanti, and Rhynchosciara sp were used and genomic sequences were amplified, sequenced and the molecular structures of the elements characterized as being putative mariner-like elements. The first group included the putative full-length elements. The second group was composed of defective mariner elements that contain a deletion overlapping most of the internal region of the transposase open reading frame. They were named Rmarl (type 1) and Rmar2 (type 2), respectively. Many conserved amino acid blocks were identified, as well as a specific D,D(34)D signature motif that was defective in some elements. Based on predicted transposase sequences, these elements
\end{abstract}


encode truncated proteins and are phylogenetically very close to mariner-like elements of the mauritiana subfamily. The inverted terminal repeat sequences that flanked the mariner-like elements are responsible for their mobility. These inverted terminal repeat sequences were identified by inverse PCR.

Key words: Rhynchosciara; Polytene chromosome; Transposon; Mariner-like element; Transposase; Inverted terminal repeat sequence

\section{INTRODUCTION}

Transposons are also called "selfish DNA" or "jumping genes"; these mobile elements copy themselves to different locus forming additional copies inside the genome. There are a variety of mobile genetic element families that belong to class II such as the mariner-like elements. These elements constitute a large fraction of the eukaryotic genome; they transpose by a "cut-and-paste" mechanism with their own transposase protein.

These elements were first identified in Drosophila mauritiana (Jacobson et al., 1986) as mariner (Mos1) elements, and were initially described as a small gene $(\sim 1.3 \mathrm{~kb})$ that encodes a single protein (mariner transposase) flanked by short sequences of inverted terminal repeats (ITRs) of $28 \mathrm{bp}$. The transposase consists of two domains; an N-terminal is the ITR binding domain and a catalytic domain in the C-terminal (Hartl et al., 1997a). The transposable mechanism is mediated by the ability of transposase to recognize the ITR sequence (Hartl et al., 1997b; Lohe et al., 1997). The two functions of the transposase, the binding in the DNA specific sequence and the excision and insertion of the transposable element, are mediated by DNA binding and catalytic domains separately. The mariner transposase contains two highly conserved motifs: WVPHEL and YSPDLAP, and the D,D(34)D signature motif (Robertson, 1993; Doak et al., 1994). The transposable process is mediated by the transposase's ability to recognize the ITR sequence. It occurs via a DNA intermediate catalyzed by the transposase with a duplication of TA nucleotides in the insertion site (Lohe et al., 1997).

Mariner transposable elements are widely distributed in nature and can be found in a variety of insects and other arthropods. Mariner family members have also been identified in various organisms such as nematodes, marine species, fungi, plants, and mammals, including humans (Robertson, 1993; Capy et al., 1996; Jarvik and Lark, 1998; Leroy et al., 2003; Mandrioli, 2003; Halaimia-Toumi et al., 2004). The mariner-like element family is subdivided into more than 13 subfamilies; the different subfamilies are present in the same genome (Robertson and MacLeod, 1993).

Rhynchosciara americana, an important and typically Brazilian model system used to study molecular and developmental biology, has became the target of new findings internationally (Santelli et al., 2004; Siviero et al., 2006; de Andrade et al., 2009). The search for a mobile element that can be used as a transforming vector could represent an important advance in understanding crucial aspects in the development of this species (Rezende-Teixeira et al., 2008a,b, 2009). In the present study, we identified two different groups of the mariner elements in the genus Rhynchosciara: the full-length and defective elements. They were called Rmar 1 or type 1 and Rmar 2 or type 2 . Some characteristics that identify the mariner-like elements and some conserved motifs were present. In situ hybridization in the salivary gland of the polytene chromosome showed a similar localization in the genus Rhynchosciara. 


\section{MATERIAL AND METHODS}

\section{Animals}

Larvae of R. americana were collected in the region of Ubatuba, State of São Paulo, Brazil, and bred in the laboratory, using the conditions established by Lara et al. (1965).

\section{PCR, inverse PCR and DNA sequencing}

Polymerase chain reaction (PCR) amplifications were made with specific primer to amplify the Ramarl (accession number: DQ784570) and Ramar2 (sccession number: DQ784571) element internal regions (pRamar1: 5'-TTTGCACAACAAGTTCAATTT-3' and 5'-TTTCTGGCAATTTACGGAT-3'; pRamar2: 5'-TTCGGTTAGCTTTCGCAGAT-3' and 5'-CTTTTCCCATCTCTCAGGCAG-3'). The protocol of DNA circularization was described in Rezende-Teixeira et al. (2008a). Primers were designed for inverse PCR (iRmar1R 5'-ATCAAAACTTCCCGCAAATG-3', iRmar1F 5'-GATGGGAAATTCTGGTTCA-3'; iRmar2R 5'-TGACGCTTACTTGGCTCAAA-3' and iRmar2F 5'-CCGTCCGATTATCACCTGTT-3') based on the internal sequences of Ramar1 and Ramar2. The PCR amplifications were performed using the Platinum Taq DNA polymerase (Invitrogen Life Technologies) according to manufacturer instructions. Cycle conditions were $94^{\circ} \mathrm{C}$ for $2 \mathrm{~min}, 35$ cycles $\left[94^{\circ} \mathrm{C}\right.$ for $30 \mathrm{~s} ; 55^{\circ} \mathrm{C}$ for $30 \mathrm{~s} ; 72^{\circ} \mathrm{C}$ for $\left.2 \mathrm{~min}\right]$ and a final extension at $72^{\circ} \mathrm{C}$ for $7 \mathrm{~min}$. The PCR products were cloned in pGemT-easy vector (Promega). Clones were sequenced using the BigDye terminator (PerkinElmer) and run on an ABI-3100 sequencer (PerkinElmer), using T7 and T3 primers. The nucleotide sequences were analyzed in a Linux workstation with Phred, Phrap Crosmatch and Consed 17 programs (Ewing and Green, 1998; Ewing et al., 1998; Gordon et al., 1998). The BLAST analyses were done in the non-redundant GenBank database (Wheeler et al., 2000). The mariner-like element sequences of Rhynchosciara have been deposited in the GenBank database and have the following accession numbers: $R$. hollaenderi Rhmar1 (GU442124) and Rhmar2 (GU442125), R. millerii Rmlmarl (GU442126) and Rmlmar2 (GU442127), R. baschanti Rbmarl (GU442122) and Rbmar2 (GU442123) and

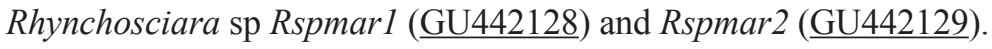

\section{In situ hybridizations}

Salivary gland chromosomes fixed with ethanol-acetic acid (3:1) were squashed in $45 \%$ acetic acid. The protocol of in situ hybridization was described in Rezende-Teixeira et al. (2008b). The preparations were analyzed in a laser scanning confocal microscope, LSM-510 (Zeiss), and positive regions were considered those labelled in most of the chromosome optical sections.

\section{RESULTS}

PCR amplifications were done using genomic DNA of different species of Rhynchosciara and primers designed from internal regions of Ramarl and Ramar2 mariner-like elements (Rezende-Teixeira et al., 2008a). The species used in this comparative study were: $R$. hollaenderi, R. millerii, R. baschanti, and Rhynchosciara sp (a species not yet described). Ini- 
tially, the amplification of the internal region was done for all species and then the flanks were identified for three of the four species studied using the inverse PCR technique. The general molecular structures of mariner elements identified in the genus Rhynchosciara are shown in Figure 1, except for $R$. baschanti, whose ITR sequences could not be identified.
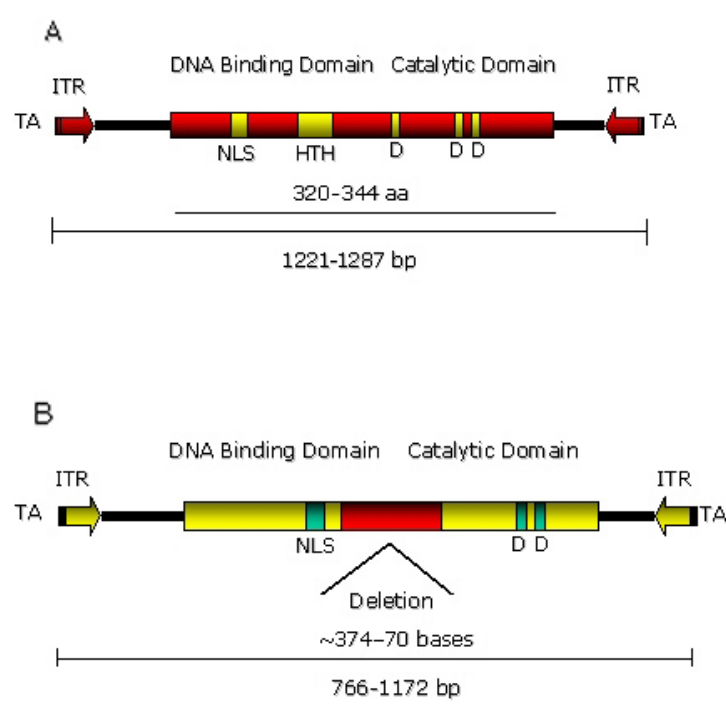

Figure 1. Molecular structure of mariner elements studied in the Rhynchosciara species. A. The putative fulllength mariner element (Rmar1). B. The putative defective mariner element (Rmar2). ITR = inverted terminal repeat sequence; $\mathrm{NLS}=$ nuclear localization signal; $\mathrm{HTH}=$ helix-turn-helix motif; aa = amino acids.

The elements were called as Rhmar 1 and Rhmar2 from R. hollaenderi, Rmlmarl and Rmlmar2 from R. millerii, Rbmar 1 and Rbmar 2 from $R$. baschanti, and Rspmarl and Rspmar 2 from Rhynchosciara sp. The consensus nucleotide sequence to elements of type 1 showed a characteristic length of 1221 to $1287 \mathrm{bp}$, corresponding to the typical size of a mariner-like element, while the elements of type 2 had a length ranging from 766 to $1172 \mathrm{bp}$. The predicted translation of the transposase open reading frame (ORF) was derived from defective copies of elements encoding 320 to 344 amino acid proteins to a type 1 element. In the elements of type 2, the deleted internal region showed a range of 374 to $70 \mathrm{bp}$, varying markedly for different species. This may be indicative that the five Rhynchosciara species, including R. americana, evolved independently.

It is possible to identify two main domains in the scheme: 1 ) in the amino-terminal region, known to contain the DNA binding domain, it is possible to identify the nuclear localization signal and the helix-turn-helix motif, responsible for the ITR binding domain, and 2) the catalytic domain is located in the carboxy-terminal, represented by the $\mathrm{D}, \mathrm{D}(34) \mathrm{D}$ catalytic triad proposed by Doak et al. (1994) and Robertson and Lampe (1995). The two main conserved motifs of the mariner transposases (WVPHEL and YSPDLAP) described by Robertson (1993) are present but with some modifications. The consensus motif identified was YVPYEL and YSPDLAP for all species. This can be an indication that this motif is specific to Rhynchosciara, and may be a selective mutation that generates this sequence that is perpetuated every generation. 
Tables 1 and 2 show the comparison between the consensus sequences of Rmarl and Rmar2 elements of different species; a high identity in nucleotide sequences is also observed. The Rmar 1 consensus transposase sequences share $63-86 \%$ amino acid identity and $73-92.7 \%$ conservative replacements, while Rmar2 transposase share $35.5-76.8 \%$ and $44-81 \%$ amino acid identity and conservative replacements.

Table 1. Comparison between the studied mariner-like element sequences amplified in the Rhynchosciara species.

\begin{tabular}{lcccc}
\hline & Ramarl & Rspmarl & Rhmarl & Rbmarl \\
\hline Rspmarl & $68.1 / 76.5(91.8)$ & - & - & - \\
Rhmarl & $80.0 / 85.2(87.4)$ & $63.1 / 73.4(88.0)$ & - & - \\
Rbmarl & $86.0 / 89.5(77.8)$ & $65.7 / 73.0(75.4)$ & $73.0 / 77.9(70.6)$ & - \\
Rmlmarl & $87.2 / 92.7(91.0)$ & $66.0 / 74.7(93.3)$ & $74.8 / 82.0(86.4)$ & $81.4 / 86.3(74.7)$ \\
\hline
\end{tabular}

Data are reported as percent of amino acid identity/conservative replacements with nucleotide identity in parentheses. For abbreviations, see Figure 4.

Table 2. Comparison between the studied mariner-like element sequences amplified in the Rhynchosciara species.

\begin{tabular}{lcccc}
\hline & Ramar2 & Rspmar2 & Rhmar2 & Rbmar2 \\
\hline Rspmar2 & $42.0 / 53.3(61.0)$ & - & - & - \\
Rhmar2 & $76.4 / 79.2(90.1)$ & $35.5 / 44.4(60.4)$ & - & - \\
Rbmar2 & $76.8 / 81.0(76.1)$ & $37.7 / 47.0(48.6)$ & $76.5 / 78.5(73.8)$ & - \\
Rmlmar2 & $63.0 / 73.0(76.0)$ & $37.5 / 44.4(55.5)$ & $61.5 / 70.0(78.6)$ & $64.1 / 73.7(60.0)$ \\
\hline
\end{tabular}

Data are reported as percent of amino acid identity/conservative replacements with nucleotide identity in parentheses. For abbreviations, see Figure 4.

The elements amplified and sequenced have a typical structure of a mariner-like element, the inverted terminal repeats with $28 \mathrm{bp}$ and TA dinucleotide immediately flanking the ITRs, as is typical for mariner insertion events (Figure 2). Only in $R$. baschanti was it not possible to identify the ITR sequence. It may suggest that these elements are fully defective and therefore the ITR sequences have been lost from the genome of this species.
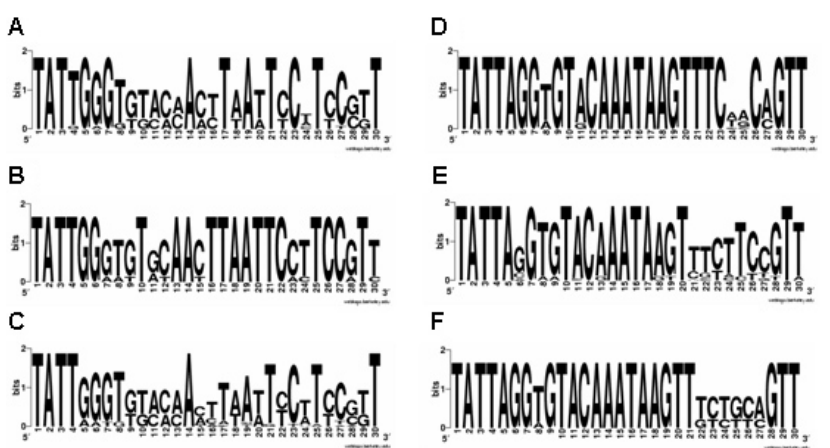

Figure 2. Sequence logo of the region flanking and inverted terminal repeats of the mariner-like elements of three Rhynchosciara species. The sequence logos were generated using the WebLogo server (http://weblogo.berkeley. edu/), and showed the consensus ITR sequence and the insertion site in genomic DNA to Rmlmarl (A) and Rmlmar2 (D); Rhmarl (B) and Rhmar2 (E); Rspmar 1 (C), and Rspmar 2 (F). The vertical axis has a maximum value of two, which is proportional to the level of sequence conservation at each position. For abbreviations, see Figure 4. 
The ITR sequences found for other species: $R$. millerii, $R$. hollaenderi and Rhynchosciara sp are highly defective. They exhibited imperfect terminal repeats with many mismatches and no specific mutation site. The opposite was observed for $R$. americana where the Ramar 1 and Ramar2 elements shared high identity (82\%) in the ITR sequences, including nine continuous nucleotides (5'-GGTGTACAA) at the 5' end and the last eight nucleotides (CTTCCGTT-3') of the sequence (Rezende-Teixeira et al., 2008a).

However, the mismatches identified in the ITR sequences of Rhynchosciara could inactivate these mariner elements by losing the capacity for mobility. Some studies show important characteristics about the ITR sequences, such as conservation of a palindrome sequence and motif mirror (Bigot et al., 2005), and a possible function, initially proposed by Pietrokovski and Henikoff (1997) and then confirmed by Augé-Gouillou et al. (2001). The ITR sequence could be a region involved in the binding of the mariner transposase by helixturn-helix motif present in the N-terminal portion. However, the conservation and evolution of ITR sequences in the mariner elements represent a puzzle to be solved.

In situ hybridizations of a mariner-like element in the salivary gland of polytene chromosomes of $R$. baschanti, $R$. hollaenderi and Rhynchosciara sp appeared as a single band. The same labeling was observed in $R$. americana using the Ramar 2 probe. The chromosomes were propidium iodide stained and the arrows showed the positive regions. The labeling appeared in the same and in a very similar region of polytene chromosome $\mathrm{A}$ in all the Rhynchosciara species studied (Figure 3).

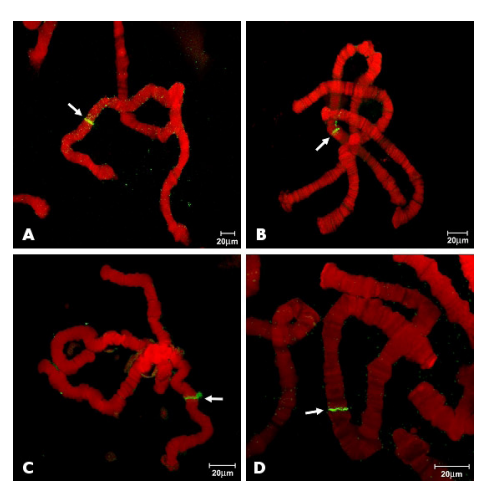

Figure 3. Laser scanner confocal microscope images of the in situ hybridization of a mariner-like element in salivary gland chromosomes using the Rhynchosciara americana mariner-like element as a probe. A. R. baschanti. B. $R$. hollaenderi. C. Rhynchosciara sp. D. R. americana.

Currently, it is known that these elements are members of a large family of transposons, which became known as mariner or mariner-like elements (Lampe et al., 1996). Over 13 subfamilies of mariner elements are known, which typically have about $40-56 \%$ nucleotide identity and $23-45 \%$ amino acid identity between the subfamilies and $25-100 \%$ amino acid identity with a particular subfamily (Robertson and MacLeod, 1993).

To investigate the phylogenetic distribution of the known full-length mariner, a phylogenetic tree based on transposase sequences was constructed. The elements used were downloaded from GenBank and the accession numbers are: $R$. americana Ramar1 (DQ784570), 
R. americana Ramar2 (DQ784571), Drosophila erecta Demar1 (U08094), Apis mellifera Ammarl (U19902), Ceratitis capitata Ccmarl (AAB17945), Caenorhabditis elegans Cemar1 (NP 497296), C. elegans Cemar2 (NP 497120), Mayetiola destructor Desmarl (AAA66077), D. mauritiana Mos1 (AAA28678), Haematobia irritans Himar1 (U11645), Chrysoperla plorabunda Cpmarl ( $\underline{\mathrm{AAA} 28265})$, Mantispa pulchella Mpmarl (U11649), Homo sapiens Hsmar2 (AAC52011), Ochlerotatus atropalpus Atmarl (Aㄴ16723), Hyalophora cecropia

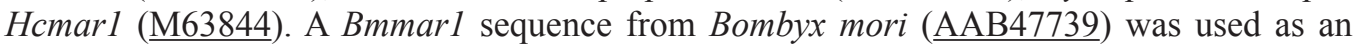
out-group (Shao and Tu, 2001). The alignment was generated by the use of ClustalX with default parameters and the tree was elaborated using a neighbor-joining algorithm and was constructed with the TreeView 1.6.6 software (Saitou and Nei, 1987; Page, 1996). The tree obtained was classified into six subfamilies (mellifera, elegans, mauritiana, irritans, cecropia, and mori) based on their branching pattern (Figure 4).

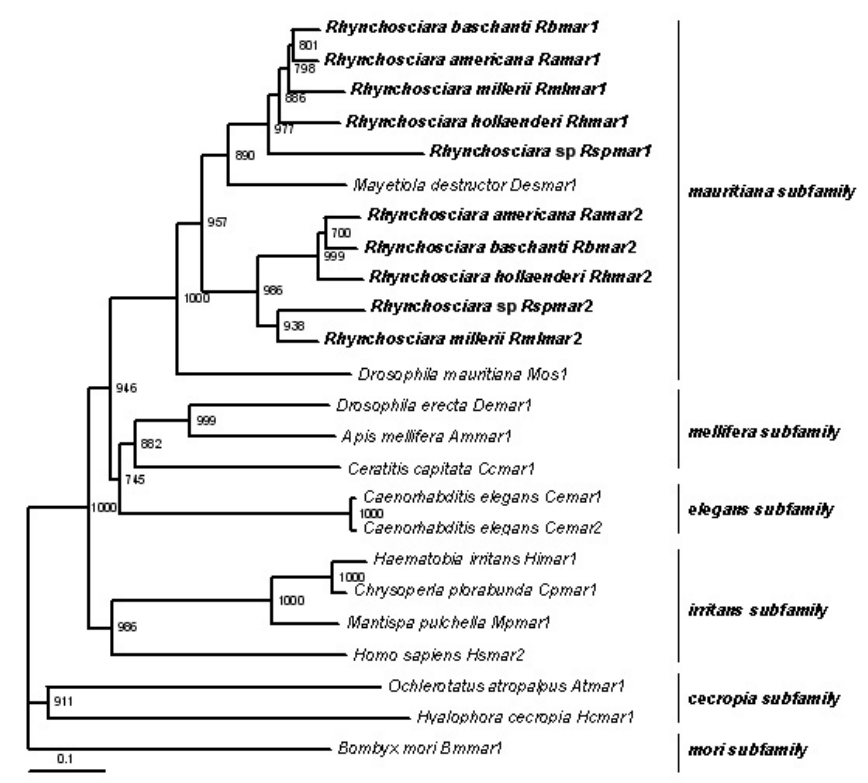

Figure 4. Phylogenetic relationship among the consensus sequences of the elements of Rhynchosciara species and other mariner elements based on their transposase amino acid sequences, using Bmmarl as an outgroup.

The grouping of mariner elements of the genus Rhynchosciara within the mauritiana subfamily was strongly supported in bootstrap analysis and amino acid identity and similarity with its sister clade. However, two different sub-clades could be identified that represented the mariner-like elements type 1 and type 2 .

\section{CONCLUSION}

The transposable elements can be autonomous or defective with respect to their own transposition. Defective elements often exhibit deletions in ORFs or in the sequence of terminal repeats necessary for its insertion. The autonomous elements are able to move non- 
functional elements, since they belong to the same subfamily. So just a functional element is necessary for the event of transposition to occur. The ability to identify an ORF for the transposase from multiple non-functional copies would indicate that the ancestor of the element Rmarl could have been a hyperactive transposase.

The characterization of mariner-like elements in the genus Rhynchosciara can be an indication that these elements populated the Rhynchosciara genome for many years. Nevertheless, these elements still represent a puzzle in the evolutionary history of this model system. Furthermore, these results represent the principle for understanding the dynamics of mariner elements and their distribution in the genome of Rhynchosciara.

\section{ACKNOWLEDGMENTS}

We would like to thank Dr. Paolo M.A. Zanotto, Juliana Velasco and Fernando Lucas Melo for sequencing support, and FAPESP and CNPq for their financial support and fellowship.

\section{REFERENCES}

Augé-Gouillou C, Hamelin MH, Demattei MV, Periquet G, et al. (2001). The ITR binding domain of the mariner Mos-1 transposase. Mol. Genet. Genomics 265: 58-65.

Bigot Y, Brillet B and Auge-Gouillou C (2005). Conservation of Palindromic and Mirror Motifs within Inverted Terminal Repeats of mariner-like elements. J. Mol. Biol. 351: 108-116.

Capy P, Vitalis R, Langin T, Higuet D, et al. (1996). Relationships between transposable elements based upon the integrase-transposase domains: is there a common ancestor? J. Mol. Evol. 42: 359-368.

de Andrade A, Siviero F, Rezende-Teixeira P, Santelli RV, et al. (2009). Molecular characterization of a putative heat shock protein cognate gene in Rhynchosciara americana. Chromosome Res. 17: 935-945.

Doak TG, Doerder FP, Jahn CL and Herrick G (1994). A proposed superfamily of transposase genes: transposon-like elements in ciliated protozoa and a common "D35E" motif. Proc. Natl. Acad. Sci. U. S. A. 91: 942-946.

Ewing B and Green P (1998). Base-calling of automated sequencer traces using Phred. II. Error probabilities. Genome Res. 8: 186-194.

Ewing B, Hillier L, Wendl MC and Green P (1998). Base-calling of automated sequencer traces using Phred. I. Accuracy assessment. Genome Res. 8: 175-185.

Gordon D, Abajian C and Green P (1998). Consed: a graphical tool for sequence finishing. Genome Res. 8: 195-202.

Halaimia-Toumi N, Casse N, Demattei MV, Renault S, et al. (2004). The GC-rich transposon Bytmarl from the deep-sea hydrothermal crab, Bythograea thermydron, may encode three transposase isoforms from a single ORF. J. Mol. Evol. 59: 747-760.

Hartl DL, Lohe AR and Lozovskaya ER (1997a). Modern thoughts on an ancient marinere: function, evolution, regulation. Ann. Rev. Genet. 31: 337-358.

Hartl DL, Lohe AR and Lozovskaya ER (1997b). Regulation of the transposable element mariner. Genetica 100: 177-184.

Jacobson JW, Medhora MM and Hartl DL (1986). Molecular structure of a somatically unstable transposable element in Drosophila. Proc. Natl. Acad. Sci. U. S. A. 83: 8684-8688.

Jarvik T and Lark KG (1998). Characterization of Soymar1, a mariner element in soybean. Genetics 149: 1569-1574.

Lampe DJ, Churchill ME and Robertson HM (1996). A purified mariner transposase is sufficient to mediate transposition in vitro. EMBO J. $15: 5470-5479$.

Lara FJS, Tamaki H and Pavan C (1965). Laboratory culture of Rhynchosciara angelae. Am. Nat. 99: 189-191.

Leroy H, Castagnone-Sereno P, Renault S, Auge-Gouillou C, et al. (2003). Characterization of Mcmarl, a mariner-like element with large inverted terminal repeats (ITRs) from the phytoparasitic nematode Meloidogyne chitwoodi. Gene 304: 35-41.

Lohe AR, De Aguiar D and Hartl DL (1997). Mutations in the mariner transposase: the D,D(35)E consensus sequence is nonfunctional. Proc. Natl. Acad. Sci. U. S. A. 94: 1293-1297.

Mandrioli M (2003). Identification and chromosomal localization of mariner-like elements in the cabbage moth Mamestra brassicae (Lepidoptera). Chromosome Res. 11: 319-322.

Page RD (1996). TreeView: an application to display phylogenetic trees on personal computers. Comput. Appl. Biosci. 
12: $357-358$

Pietrokovski S and Henikoff S (1997). A helix-turn-helix DNA-binding motif predicted for transposases of DNA transposons. Mol. Gen. Genet. 254: 689-695.

Rezende-Teixeira P, Siviero F, Andrade A, Santelli RV, et al. (2008a). Mariner-like elements in Rhynchosciara americana (Sciaridae) genome: molecular and cytological aspects. Genetica 133: 137-145.

Rezende-Teixeira P, Siviero F, Brandao AS, Santelli RV, et al. (2008b). Molecular characterization of a retrotransposon in the Rhynchosciara americana genome and its association with telomere. Chromosome Res. 16: 729-742.

Rezende-Teixeira P, Siviero F, da Costa RM and Machado-Santelli GM (2009). The R2 mobile element of Rhynchosciara americana: molecular, cytological and dynamic aspects. Chromosome Res. 17: 455-467.

Robertson HM (1993). The mariner transposable element is widespread in insects. Nature 362: 241-245.

Robertson HM and MacLeod EG (1993). Five major subfamilies of mariner transposable elements in insects, including the Mediterranean fruit fly, and related arthropods. Insect Mol. Biol. 2: 125-139.

Robertson HM and Lampe DJ (1995). Distribution of transposable elements in arthropods. Annu. Rev. Entomol. 40: 333-357.

Saitou N and Nei M (1987). The neighbor-joining method: a new method for reconstructing phylogenetic trees. Mol. Biol. Evol. 4: 406-425.

Santelli RV, Siviero F, Machado-Santelli GM, Lara FJ, et al. (2004). Molecular characterization of the B-2 DNA puff gene of Rhynchosciara americana. Chromosoma 113: 167-176.

Shao H and Tu Z (2001). Expanding the diversity of the IS630-Tc1-mariner superfamily: discovery of a unique DD37E transposon and reclassification of the DD37D and DD39D transposons. Genetics 159: 1103-1115.

Siviero F, Rezende-Teixeira P, Andrade A, Machado-Santelli GM, et al. (2006). Analysis of expressed sequence tags from Rhynchosciara americana salivary glands. Insect Mol. Biol. 15: 109-118.

Wheeler DL, Chappey C, Lash AE, Leipe DD, et al. (2000). Database resources of the National Center for Biotechnology Information. Nucleic Acids Res. 28: 10-14. 